\title{
SOME HILBERT SPACES OF ENTIRE FUNCTIONS
}

\section{LOUIS DE BRANGES ${ }^{1}$}

Communicated by R. P. Boas, September 12, 1960

If $E(z)$ is an entire function such that

(1)

$$
|E(\bar{z})|<|E(z)|
$$

for $y>0(z=x+i y)$, we write

$$
E(z)=A(z)-i B(z)
$$

where $A(z)$ and $B(z)$ are entire functions which are real for real $z$, and

$$
K(w, z)=[B(z) \bar{A}(w)-A(z) \bar{B}(w)] /[\pi(z-\bar{w})] .
$$

Let $\mathfrak{F C}(E)$ be the Hilbert space of entire functions $F(z)$ such that

$$
\|F\|^{2}=\int|F(t)|^{2}|E(t)|^{-2} d t<\infty
$$

with integration on the real axis, and

$$
|F(z)|^{2} \leqq\|F\|^{2} K(z, z)
$$

for all complex $z$. This space was introduced in [7] and characterized axiomatically. For each complex number $w, K(w, z)$ belongs to $\mathfrak{H C}(E)$ as a function of $z$ and

$$
F(w)=\langle F(t), K(w, t)\rangle
$$

for each $F(z)$ in $\mathfrak{H}(E)$.

The typical example of such a space occurs in Fourier analysis, and then there is a family $(E(a, z))$ of entire functions satisfying (1), $a>0$ :

$$
\begin{gathered}
E(a, z)=\exp (-i a z), \\
A(a, z)=\cos (a z), \quad B(a, z)=\sin (a z) .
\end{gathered}
$$

When $a \leqq b, \mathfrak{F}(E(a))$ is contained isometrically in $\mathfrak{F}(E(b))$, and every one of these spaces is contained isometrically in $L^{2}(-\infty,+\infty)$. A necessary and sufficient condition that an entire function $F(z)$ belong to $\mathfrak{F C}(E(a))$ is that it be of the form

$$
\pi F(z)=\int f(t) \cos (t z) d t+\int g(t) \sin (t z) d t,
$$

1 This research was supported by the United States Air Force under contract No. AF49(638)-253. 
where $f(t)$ and $g(t)$ are measurable complex valued functions of $t \geqq 0$, which are square integrable and which vanish a.e. outside of $[0, a]$. In this case,

$$
\pi \int|F(t)|^{2} d t=\int|f(t)|^{2} d t+\int|g(t)|^{2} d t .
$$

There is a closely analogous representation for the most general space $\mathcal{F}(E)$. For simplicity of presentation, we will suppose that $E(z)$ has no real zeros and that $E(0)=1$.

Let

$$
m(t)=\left(\begin{array}{ll}
\alpha(t) & \beta(t) \\
\beta(t) & \gamma(t)
\end{array}\right)
$$

be a matrix valued function of $t>0$, where $\alpha(t), \beta(t), \gamma(t)$ are real valued, absolutely continuous functions of $t>0$ such that

$$
\alpha^{\prime}(t) \geqq 0, \quad \gamma^{\prime}(t) \geqq 0, \quad \beta^{\prime}(t)^{2} \leqq \alpha^{\prime}(t) \gamma^{\prime}(t)
$$

a.e. for $t>0$,

$$
\alpha(t)>0 \text { for } t>0 \text { and } \lim _{t \searrow 0} \alpha(t)=0,
$$

and

$$
\lim _{t \rightarrow \infty}[\alpha(t)+\gamma(t)]=\infty .
$$

A real number $b>0$ is said to be singular with respect to $m(t)$ if it belongs to an open interval $(a, c)$ in which $\alpha^{\prime}(t), \beta^{\prime}(t), \gamma^{\prime}(t)$ are equal a.e. to a constant multiple of a single function and

$$
\beta^{\prime}(t)^{2}=\alpha^{\prime}(t) \gamma^{\prime}(t)
$$

a.e. Otherwise, $t$ is said to be regular with respect to $m(t)$. Let $L^{2}(m)$ be the Hilbert space of (equivalence classes of) pairs $(f(t), g(t))$ of measurable complex valued functions of $t \geqq 0$ such that

$$
\|(f, g)\|^{2}=\int(f(t), g(t)) d m(t)(f(t), g(t))^{-}<\infty .
$$

Here,

$$
(f(t), g(t))^{-}=\left(\begin{array}{l}
\bar{f}(t) \\
\bar{g}(t)
\end{array}\right)
$$

and the integral is to be interpreted as 


$$
\int(f(t), g(t)) m^{\prime}(t)(f(t), g(t))-d t .
$$

By the equivalence of $\left(f_{1}(t), g_{1}(t)\right)$ and $\left(f_{2}(t), g_{2}(t)\right)$ in an interval $(a, b)$, we mean that

$$
\int_{a}^{b}(f(t), g(t)) d m(t)(f(t), g(t))^{-}=0
$$

where

$$
(f(t), g(t))=\left(f_{2}(t), g_{2}(t)\right)-\left(f_{1}(t), g_{1}(t)\right) .
$$

Let $L_{0}^{2}(m)$ be the closed subspace of $L^{2}(m)$ formed by the elements $(f, g)$ of $L^{2}(m)$ such that $(f(t), g(t))$ is equivalent to a constant in any interval $(a, b)$ containing only singular points with respect to $m(t)$. Since $\beta(t)$ is real valued by hypothesis, $(\bar{f}(t), \bar{g}(t))$ belongs to $L^{2}(m)$ whenever $(f(t), g(t))$ belongs to $L^{2}(m)$ and these elements have the same norm; if one belongs to $L_{0}^{2}(m)$, so does the other. Let $H$ be the transformation in $L_{0}^{2}(m)$ defined by

$$
\left(f_{2}, g_{2}\right)=H\left(f_{1}, g_{1}\right)
$$

if $\left(f_{1}, g_{1}\right)$ and $\left(f_{2}, g_{2}\right)$ are elements of $L_{0}^{2}(m)$, and $\left(f_{1}, g_{1}\right)$ is (equivalent to a pair of functions) such that $f_{1}(t)$ is continuous for $t>0, g_{1}(t)$ is continuous for $t \geqq 0, g_{1}(0)=0$, and

$$
\left(f_{1}(b), g_{1}(b)\right) I-\left(f_{1}(a), g_{1}(a)\right) I=\int_{a}^{b}\left(f_{2}(t), g_{2}(t)\right) d m(t)
$$

whenever $0<a<b<\infty$. Here

$$
I=\left(\begin{array}{rr}
0 & -1 \\
1 & 0
\end{array}\right)
$$

and (5) is to be taken in the sense that

$$
\begin{aligned}
& g_{1}(b)-g_{1}(a)=\int_{a}^{b}\left[f_{2}(t) \alpha^{\prime}(t)+g_{2}(t) \beta^{\prime}(t)\right] d t, \\
& f_{1}(a)-f_{1}(b)=\int_{a}^{b}\left[f_{2}(t) \beta^{\prime}(t)+g_{2}(t) \gamma^{\prime}(t)\right] d t .
\end{aligned}
$$

Both integrals are absolutely convergent by the Schwarz inequality, using (2) and the finiteness of $\left\|\left(f_{2}, g_{2}\right)\right\|$. The transformation $H$ is welldefined because the step functions which belong to $L^{2}(m)$ are dense in $L^{2}(m)$. Note that if $\left(f_{1}, g_{1}\right)$ is in the domain of $H$, then so is $\left(\bar{f}_{1}, \bar{g}_{1}\right)$; if $\left(f_{2}, g_{2}\right)=H\left(f_{1}, g_{1}\right)$, then $\left(\bar{f}_{2}, \bar{g}_{2}\right)=H\left(\bar{f}_{1}, \bar{g}_{1}\right)$. 
By a Hilbert space, we mean a complete inner product space over the complex numbers, with no restriction on dimension. The spaces which actually occur are separable, but they may have finite dimension. Our transformations are linear, but they need not be everywhere defined or bounded.

TheOREM I. Let $m(t)$ be a matrix valued function of $t>0$ which satisfies (2), (3), and (4). Suppose that there exists a family $(E(t, z))$ of entire functions satisfying (1) with no real zeros, such that $E(t, 0)=1$, $t>0$, such that for each complex number $w, E(t, w)$ is a continuous function of $t>0$,

$$
(A(b, w), B(b, w)) I-(A(a, w), B(a, w)) I
$$

$$
=w \int_{a}^{b}(A(t, w), B(t, w)) d m(t)
$$

whenever $0<a<b<\infty$, and

$$
\lim _{a \searrow 0} K(a, w, w)=0 .
$$

Then, when $a<b$ are regular points with respect to $m(t), \mathfrak{F}(E(a))$ is contained isometrically in $3(E(b))$. For all nonreal complex w,

$$
\lim _{b \rightarrow \infty} K(b, w, w)=\infty .
$$

There is a unique non-negative measure $\mu$ on the Borel sets of the real line such that

$$
\int\left(1+t^{2}\right)^{-1}|E(a, t)|^{2} d \mu(t)<\infty
$$

for each regular $a>0$, and

$$
\frac{y}{\pi} \int \frac{|E(a, t)|^{2} d \mu(t)}{(t-x)^{2}+y^{2}}=\lim _{b \rightarrow \infty} \frac{y}{\pi} \int \frac{|E(a, t)|^{2}|E(b, t)|^{-2} d t}{(t-x)^{2}+y^{2}}
$$

for $y>0$. When $a>0$ is regular with respect to $m(t), \mathfrak{H C}(E(a))$ is contained isometrically in $L^{2}(\mu)$. The union of the spaces $\mathfrak{H C}(E(a))$, with a regular, is dense in $L^{2}(\mu)$.

THEOREM II. Let $E(z)$ be an entire function which satisfies (1) and has no real zeros, such that $E(0)=1$. Let $\nu$ be a non-negative measure on the Borel sets of the real line such that $\mathfrak{H C}(E)$ is contained isometrically in $L^{2}(\nu)$. Then, $E(z)=E(c, z)$ and $\nu=\mu$ for some choice of $m(t)$ and $E(t, z)$ as in Theorem I, and some $c>0$ which is regular with respect to $m(t)$. 
Let $\chi(a, t)$ be the function which is equal to 1 when $t \leqq a$ and equal to 0 when $t>a$.

Theorem III. Let $m(t)$ and $E(t, z)$ be as in Theorem I.

(A) Let $c>0$ be regular with respect to $m(t)$. Then,

$$
\chi(c, t)(A(t, w), B(t, w))
$$

belongs to $L_{0}^{2}(m)$ for every complex number w. For each element $(f, g)$ of $L_{0}^{2}(m)$ which is equivalent to zero outside of $[0, c]$, define a corresponding "eigentransform" $F(z)$ by

$$
\pi F(w)=\int(f(t), g(t)) d m(t)(A(t, \bar{w}), B(t, \bar{w}))^{-}
$$

for all complex w. Then, $F(z)$ is an entire function, it belongs to $\mathfrak{H}(E(c))$, and

$$
\pi \int|F(t)|^{2} d \mu(t)=\int(f(t), g(t)) d m(t)(f(t), g(t))^{-} .
$$

If $G(z)$ is in $\operatorname{FC}(E(c))$, then $G(z)$ is equal to $F(z)$ for some such choice of $(f, g)$. If $F(z)$ is the eigentransform of $(f, g)$, then $F^{*}(z)=\bar{F}(\bar{z})$ is the eigentransform of $(\bar{f}, \bar{g})$.

(B) Let $c>0$ be regular with respect to $m(t)$. Let $\left(f_{1}, g_{1}\right)$ be an element of $L_{0}^{2}(m)$ which is equivalent to zero outside of $[0, c]$, and let $F_{1}(z)$ be the corresponding eigentransform. A necessary and sufficient condition that $\left(f_{1}, g_{1}\right)$ be in the domain of $H$ is that $z F_{1}(z)$ belong to $\operatorname{HC}(E(c))$. In this case, let $\left(f_{2}, g_{2}\right)=H\left(f_{1}, g_{1}\right)$ and let $F_{2}(z)$ be the corresponding eigentransform. Then, $F_{2}(z)=z F_{1}(z)$ for all complex $z$.

(C) If $(f, g)$ is in $L_{0}^{2}(m)$, the corresponding eigentransform $F(x)$ is defined by

$$
F(x)=\lim _{c \rightarrow \infty} \int_{0}^{c}(f(t), g(t)) d m(t)(A(t, x), B(t, x))^{-}
$$

This limit exists in the metric of $L^{2}(\mu)$ and (10) holds. Every element $G(x)$ of $L^{2}(\mu)$ is equal, a.e. with respect to $\mu$, to the eigentransform $F(x)$ of an element $(f, g)$ of $L_{0}^{2}(m)$. If $F(x)$ is the eigentransform of $(f, g)$, then $\bar{F}(x)$ is the eigentransform of $(\bar{f}, \bar{g})$.

(D) Let $\left(f_{1}, g_{1}\right)$ be an element of $L_{0}^{2}(m)$ and let $F_{1}(x)$ be the corresponding eigentransform. A necessary and sufficient condition that $\left(f_{1}, g_{1}\right)$ be in the domain of $H$ is that $x F_{1}(x)$ be in $L^{2}(\mu)$. In this case, let $\left(f_{2}, g_{2}\right)=H\left(f_{1}, g_{1}\right)$ and let $F_{2}(x)$ be the corresponding eigentransform. Then, $F_{2}(x)=x F_{1}(x)$ a.e. with respect to $\mu$. 


\section{REFERENCES}

1. R. P. Boas, Jr., Entire functions, New York, Academic Press, 1954.

2. L. de Branges, Local operators on Fourier transforms, Duke Math. J. vol. 25 (1958) pp. 143-154.

3. - The a-local operator problem, Canad. J. Math. vol. 11 (1959) pp. $583-592$.

4. - The Stone-Weierstrass theorem, Proc. Amer. Math. Soc. vol. 10 (1959) pp. 822-824. 832.

5. — The Bernstein problem, Proc. Amer. Math. Soc. vol. 10 (1959) pp. 825-

6. - Some mean squares of entire functions, Proc. Amer. Math. Soc. vol. 10 (1959) pp. 833-839.

7. - Some Hilbert spaces of entire functions, Proc. Amer. Math. Soc. vol. 10 (1959) pp. 840-846.

8. - Some Hilbert spaces of entire functions, Trans. Amer. Math. Soc. vol. 96 (1960) pp. 259-295. Soc.

9. - Some Hilbert spaces of entire functions, submitted to Trans. Amer. Math.

10. M. H. Stone, Linear transformations in Hilbert space and their applications to analysis, Amer. Math. Soc. Colloquium Publications, vol. 15, 1932.

The Institute for Advanced StUdy 